OPEN ACCESS

Edited by:

Wen-Qing Li,

Peking University Cancer Hospital,

China

Reviewed by:

Yun Hak Kim,

Pusan National University,

South Korea

Ioana Cosgarea,

Newcastle University, United Kingdom

*Correspondence:

Mengyun Wang

wangmengyun@fudan.edu.cn

${ }^{\text {t}}$ These authors have contributed equally to this work

Specialty section: This article was submitted to

Skin Cancer,

a section of the journal

Frontiers in Oncology

Received: 24 December 2020

Accepted: 29 January 2021

Published: 24 March 2021

Citation:

Tong X, Qu X and Wang M (2021) A Four-Gene-Based Prognostic Model Predicts Overall Survival in Patients With Cutaneous Melanoma.

Front. Oncol. 11:639874.

doi: 10.3389/fonc.2021.639874

\section{A Four-Gene-Based Prognostic Model Predicts Overall Survival in Patients With Cutaneous Melanoma}

\author{
Xiaoxia Tong ${ }^{1,2 \dagger}$, Xiaofei $\mathrm{Qu}^{1,2 \dagger}$ and Mengyun Wang ${ }^{1,2 *}$ \\ ${ }^{1}$ Cancer Institute, Fudan University Shanghai Cancer Center, Shanghai, China, ${ }^{2}$ Department of Oncology, Shanghai Medical \\ College, Fudan University, Shanghai, China
}

Background: Cutaneous melanoma $(\mathrm{CM})$ is one of the most aggressive cancers with highly metastatic ability. To make things worse, there are limited effective therapies to treat advanced CM. Our study aimed to investigate new biomarkers for CM prognosis and establish a novel risk score system in CM.

Methods: Gene expression data of CM from Gene Expression Omnibus (GEO) datasets were downloaded and analyzed to identify differentially expressed genes (DEGs). The overlapped DEGs were then verified for prognosis analysis by univariate and multivariate COX regression in The Cancer Genome Atlas (TCGA) datasets. Based on the gene signature of multiple survival associated DEGs, a risk score model was established, and its prognostic and predictive role was estimated through Kaplan-Meier (K-M) analysis and log-rank test. Furthermore, the correlations between prognosis related genes expression and immune infiltrates were analyzed via Tumor Immune Estimation Resource (TIMER) site.

Results: A total of 103 DEGs were obtained based on GEO cohorts, and four genes were verified in TCGA datasets. Subsequently, four genes (ADAMDEC1, GNLY, HSPA13, and TRIM29) model was developed by univariate and multivariate Cox regression analyses. The K-M plots showed that the high-risk group was associated with shortened survival than that in the low-risk group $(P<0.0001)$. Multivariate analysis suggested that the model was an independent prognostic factor (high-risk vs. low-risk, $\mathrm{HR}=2.06, P<0.001$ ). Meanwhile, the high-risk group was prone to have larger breslow depth $(P<0.001)$ and ulceration $(P<0.001)$.

Conclusions: The four-gene risk score model functions well in predicting the prognosis and treatment response in $\mathrm{CM}$ and will be useful for guiding therapeutic strategies for $\mathrm{CM}$ patients. Additional clinical trials are needed to verify our findings.

Keywords: prognosis, cutaneous melanoma, risk score, gene signature, survival 


\section{INTRODUCTION}

Cutaneous melanoma (CM) accounts for over $74 \%$ of skin cancer related death each year (1), which makes it one of the most malignant cancers, with tremendously poor prognosis $(2,3)$. The incidence of $\mathrm{CM}$ has continued to increase annually. Although tremendous efforts toward early detection and therapeutics were made, advanced stage melanoma patients still exhibit disappointing prognosis with 5-year overall survival rate ranging from $45 \%$ for stage III to $18 \%$ for stage IV $(4,5)$.

Cutaneous melanoma is a highly heterogeneous tumor, in terms of clinical and complicated molecular (5). Several clinical features, such as age, gender, stage, ulceration and breslow thickness have been shown to be the important clinicopathological characteristics for predicting the outcome of CM patient (6). However, due to the high potentiality for CM metastasis, the prognosis remains poor. Molecular biomarkers are important in guiding treatment selection and predicting outcome in tumor patients (7-9). For example, the 21gene recurrence score assay is prognostic for women with nodenegative, estrogen-receptor-positive breast cancer treated with tamoxifen (10). Although hundreds of studies have explored the prognostic value of molecular markers, there is still no recommended molecular marker to predict CM prognosis.

In the current study, we were devoted to exploring new biomarkers and establishing a risk score model to predict prognosis, aiming to provide appropriate therapeutic methods for CM patients.

\section{MATERIALS AND METHODS}

\section{Gene Expression Omnibus (GEO) Datasets Collection and Enrichment Analysis}

Gene expression raw microarray cell intensity (CEL) profiles of $\mathrm{CM}$ were evaluated in three independent datasets from the GEO database (accession number: GSE7553, GSE46517, and GSE15605), which included 57 tumor tissue samples and three normal skin samples; 85 tumor tissue samples and eight normal skin samples; 60 tumor tissue samples and six normal skin samples, respectively. The microarray data GSE65904 containing 214 patients was downloaded to verify our risk model. Four patients were deleted due to lack of follow-up information. When more than one probe matched the same gene ID, the mean expression value of the gene was used for our study.

\section{The Cancer Genome Atlas (TCGA) Dataset}

The TCGA CM dataset, containing 459 tumor samples which included raw counts of RNAseq expression data and clinicopathological characteristics were obtained from cBioPortal website. The TCGA dataset was randomly divided into two parts: the training cohort and the validation cohort.

\section{Identification of Common Differential Expression Genes (DEG)}

The GSE7553, GSE46517, and GSE15605 expression profiles were normalized and the DEG were calculated using the LIMMA package. In this study, Gene sets with False Discovery Rate $(\mathrm{FDR})<0.05$ and with the threshold of $|\log \mathrm{FC}|>1$ were defined as DEGs. All the data processing and normalization were performed using the R software.

\section{Identification and Selection of Prognosis- Related Genes}

Univariate and multivariate Cox regression analyses model were commonly employed in survival analysis. Genes were considered significant when the $P$ value were $<0.05$ in the univariate and multivariate Cox regression analysis based on training and validation cohorts. These genes were used to construct the risk model. The fitness of the models was compared based on Akaike information criterion (AIC) and the lowest value of AIC provided the sensitivity and specificity. Subsequently, four genes (ADAMDEC1, GNLY, HSPA13, and TRIM29) were selected.

\section{Construction and Assessment of Risk Score System}

Based on the prognosis associated genes, a risk score model was constructed for the CM patients. Each gene was added one at a time in the risk score system and the risk score for each patient was calculated as the sum of each gene's score as follows:

Risk score $=\beta$ gene $1^{*}$ Exp gene $1+\beta$ gene $2^{*}$ Exp gene $2+\ldots$

$$
+\beta \text { gene }(n)^{*} \operatorname{Exp} \text { gene }(\mathrm{n})
$$

In this formula, $\beta$ gene(n) represents the coefficient of each gene from univariate Cox regression analysis, and Exp gene(n) displays the expression of each gene.

Then all TCGA patients were separated into high and lowrisk subgroups according to the optimal cut-off value of risk score. The optimal cut-off value of risk score was determined by the time-dependent receiver operating characteristic (ROC) curve using "survivalROC" package. To compare the survival time difference between the low- and high-risk group, K-M curve was produced by the "Survminer" package using the log-rank test. The predictive accuracy of this risk score model was determined by time-dependent ROC curve analysis. The area under the curve (AUC) was calculated to measure the predictive ability of the gene signature for clinical outcomes.

\section{Immune Infiltration Analysis}

The abundance of tumor infiltrating immune cells in CM was predicted using the Tumor Immune Estimation Resource (TIMER) algorithm. The correlation between prognostic gene expression and the abundance of different immune cells, including CD8+ T cells, CD4+ T cells, macrophages, B cells, neutrophils, and dendritic cells was measured using the 
Spearman's test. All hypothetical tests were two-sided and $P$ values $<0.05$ were considered statistically significant.

\section{RESULTS}

\section{Screening of DEG}

To describe our study more clearly, a flow chart of the analysis procedure was developed (Figure 1). After the analyses of GSE7553, GSE46517, and GSE15605 data sets, DEGs were identified and selected. The overlap among three data sets included 103 DEGs was shown in the Venn diagram (Figure 2A). The volcano plots and heatmap of each data set are shown in Figures 2B-G.

\section{Construction of Risk Score System}

We conducted univariate and multivariate Cox regression to investigate the correlation of the DEGs with the overall survival of TCGA CM patients in training, validation and total cohort. Basic characteristics of the patients are shown in Table 1. The result revealed that GNLY, DFNA28, ADAMDEC1, ALOXE3, EFNA3, EPN3, EVPL, FERMT1, HSPA13, JAG2, RAPGEFL1, SULT2B1, TGM3, and TRIM29 were significant prognostic factors. Furthermore, in order to select the best performance efficacy predictive model with the lowest AIC value, we performed the stepwise multivariate Cox regression analysis to identify independent predictors for overall survival of total TCGA CM patients. Finally, four prognosis-associated genes (GNLY, ADAMDEC1, HSPA13, and TRIM29) were selected for constructing the risk score system (Table 2). The formula was as follows:

Riskscore $=(-0.101)^{*} \operatorname{ExpADAMDEC1}+(-0.091)^{*} \operatorname{Exp} G N L Y+$

$$
(-0.284)^{*} \operatorname{ExpHSPA} 13+0.102 * \operatorname{ExpTRIM} 29
$$

To evaluate the prognostic significance of the risk score, $\mathrm{K}-\mathrm{M}$ plot of high and low risk CM patients were conducted. According to the optimal cut-off value of risk score, the patients in the total TCGA cohort were classified into high (312 patients) and low (147 patients) risk groups. Compared to the high-risk group with the median OS time of 27.76 months,

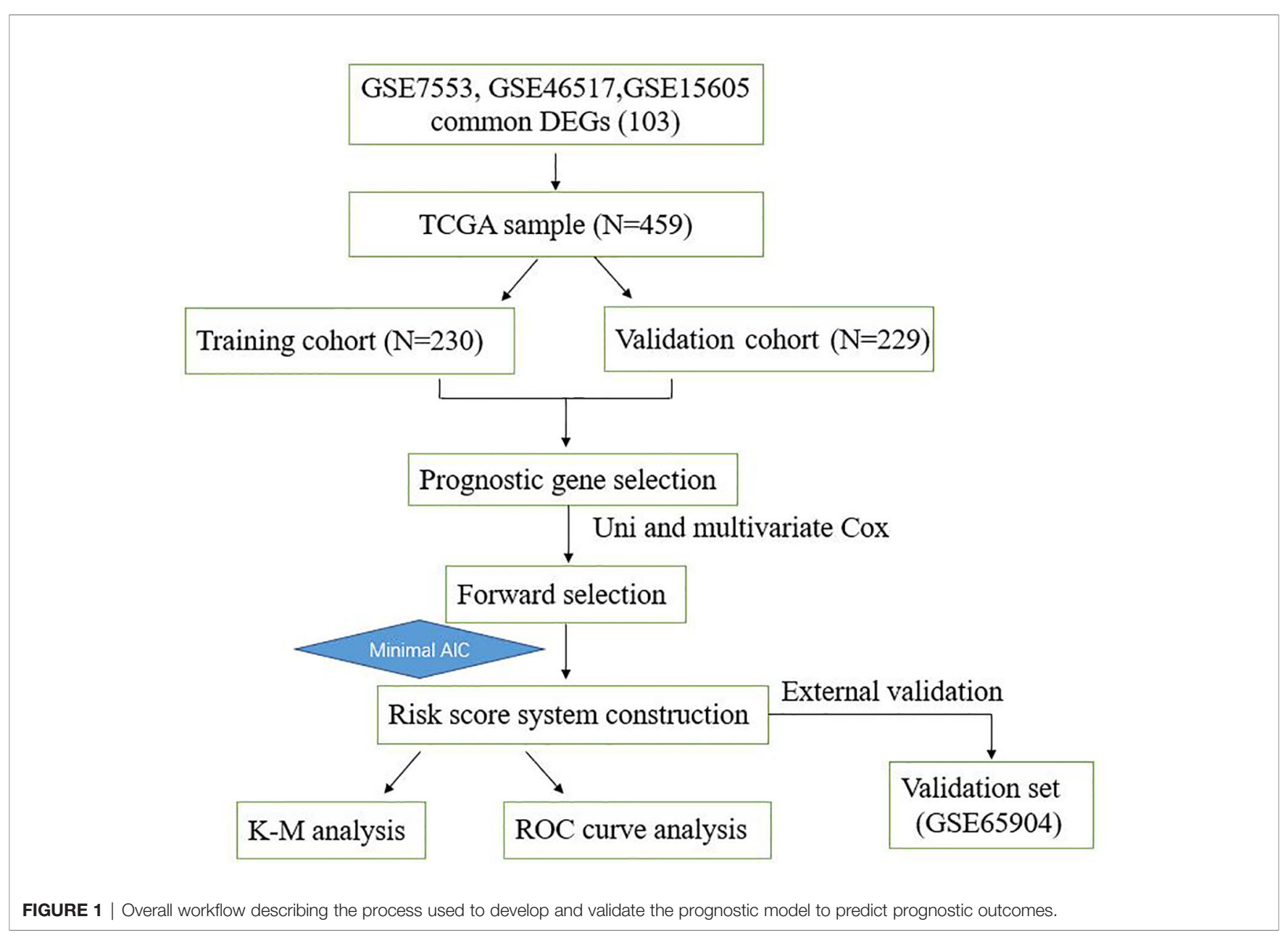


A

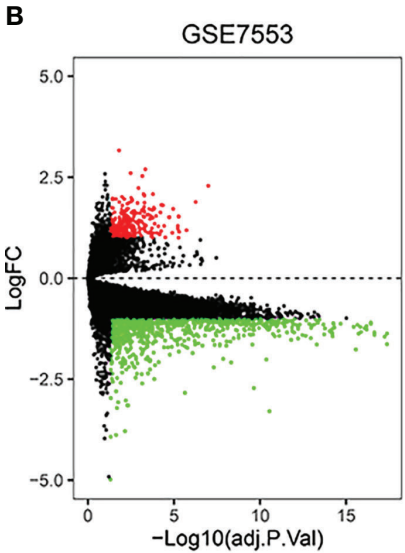

E

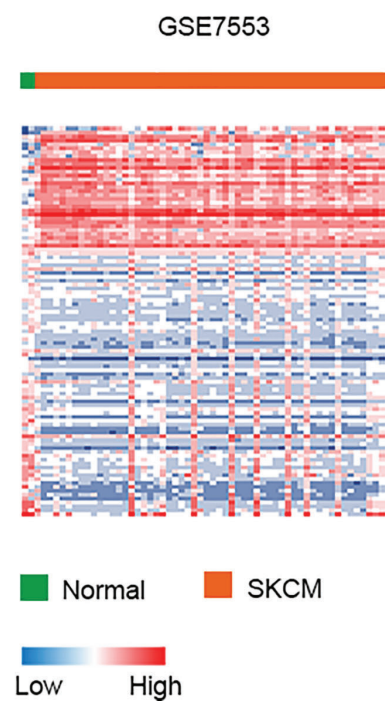

GSE7553 GSE5605

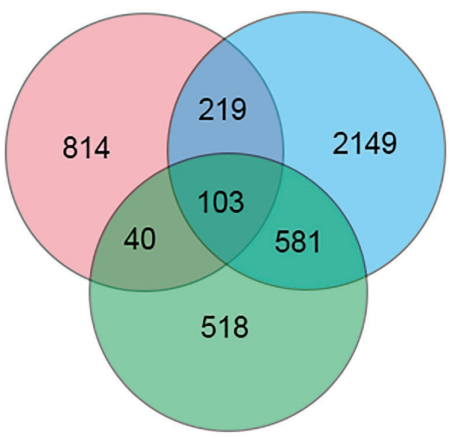

GSE46501

C

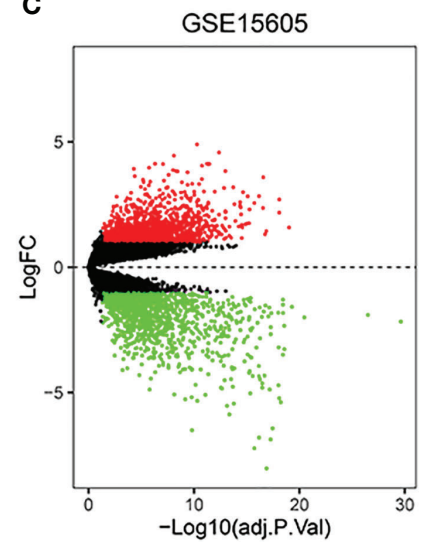

$\mathbf{F}$

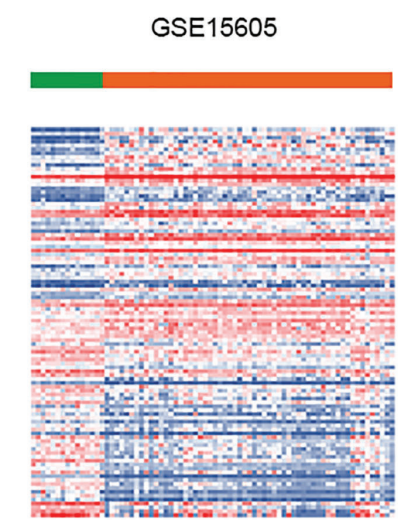

D

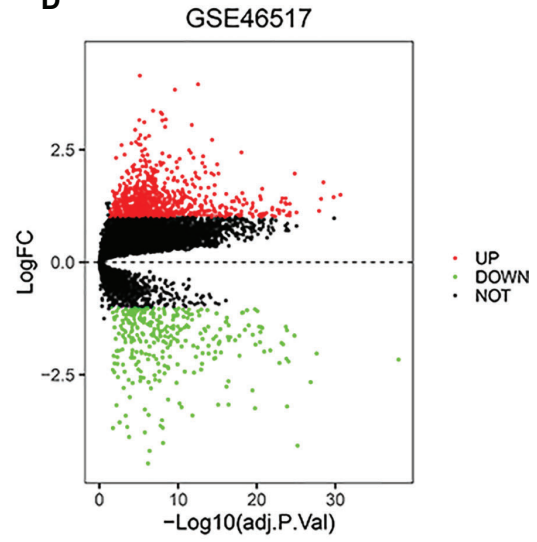

G

GSE46517

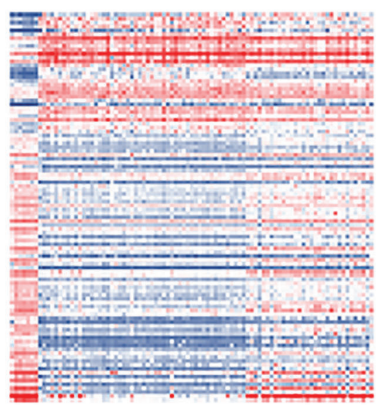

FIGURE 2 | DEGs in three data sets. (A) Venn diagram of DEGs. (B-D) The volcano plots visualize the DEGs in GSE7553, GSE15605, and GSE46517, respectively. The red nodes represent upregulated genes. The green nodes represent downregulated genes. (E-G) Heatmap of the top $103 \mathrm{DEGs}$ according to the value of $|\log F C|>1$ and FDR $<0.05$. The color in heat maps from blue to red shows the progression from low expression to high expression. logFC, log fold change. 
TABLE 1 | Basic characteristics of TCGA CM patients.

\begin{tabular}{|c|c|c|c|c|c|c|c|}
\hline \multirow[t]{2}{*}{ Characteristics } & \multirow[t]{2}{*}{ Groups } & \multicolumn{2}{|c|}{ Total $(\mathrm{N}=459)$} & \multicolumn{2}{|c|}{ Training cohort $(\mathrm{N}=229)$} & \multicolumn{2}{|c|}{ Validation cohort $(\mathrm{N}=\mathbf{2 3 0})$} \\
\hline & & No & $\%$ & No & $\%$ & No & $\%$ \\
\hline \multirow[t]{2}{*}{ Age } & $\leq 58$ & 233 & 50.7 & 116 & 50.7 & 117 & 50.9 \\
\hline & $>58$ & 226 & 49.3 & 113 & 49.3 & 113 & 49.1 \\
\hline \multirow[t]{2}{*}{ Sex } & Female & 175 & 38.1 & 82 & 35.8 & 93 & 40.4 \\
\hline & Male & 284 & 61.9 & 147 & 64.2 & 137 & 59.6 \\
\hline \multirow[t]{3}{*}{ Metastasis } & No & 410 & 89.3 & 209 & 91.3 & 201 & 87.4 \\
\hline & Yes & 23 & 5 & 9 & 3.9 & 14 & 6.1 \\
\hline & missing & 26 & 5.7 & 11 & 4.8 & 15 & 6.5 \\
\hline \multirow[t]{3}{*}{ Ulceration } & No & 145 & 31.6 & 70 & 30.6 & 75 & 32.6 \\
\hline & Yes & 165 & 35.9 & 92 & 40.2 & 73 & 31.7 \\
\hline & Missing & 149 & 32.5 & 67 & 29.2 & 82 & 35.7 \\
\hline \multirow[t]{6}{*}{ Pathologic Stage } & 0 & 6 & 1.3 & 3 & 1.3 & 3 & 1.3 \\
\hline & I & 77 & 16.8 & 40 & 17.5 & 37 & 16.1 \\
\hline & $\|$ & 139 & 30.3 & 73 & 31.8 & 66 & 28.7 \\
\hline & III & 169 & 36.8 & 81 & 35.4 & 88 & 38.3 \\
\hline & IV & 22 & 4.8 & 9 & 4 & 13 & 5.6 \\
\hline & Missing & 46 & 10 & 23 & 10 & 23 & 10 \\
\hline \multirow[t]{4}{*}{ Tumor Site } & Trunk & 166 & 36.2 & 86 & 37.6 & 80 & 34.8 \\
\hline & Extremities & 194 & 42.3 & 100 & 43.7 & 94 & 40.9 \\
\hline & Head and neck & 35 & 7.6 & 15 & 6.5 & 20 & 8.7 \\
\hline & Missing & 64 & 13.9 & 28 & 12.2 & 36 & 15.6 \\
\hline \multirow[t]{4}{*}{ Breslow thickness (mm) } & $\leq 2$ & 136 & 29.6 & 73 & 31.9 & 63 & 27.4 \\
\hline & $2-5$ & 113 & 24.6 & 52 & 22.7 & 61 & 26.5 \\
\hline & $>5$ & 105 & 22.9 & 59 & 25.8 & 46 & 20 \\
\hline & Missing & 105 & 22.9 & 45 & 19.6 & 60 & 26.1 \\
\hline \multirow[t]{3}{*}{ Chemotherapy } & No & 323 & 70.4 & 153 & 66.8 & 170 & 73.9 \\
\hline & Yes & 88 & 19.2 & 49 & 21.4 & 39 & 17 \\
\hline & Missing & 48 & 10.4 & 27 & 11.8 & 21 & 9.1 \\
\hline \multirow[t]{3}{*}{ Radiotherapy } & No & 341 & 74.3 & 170 & 74.2 & 171 & 74.3 \\
\hline & Yes & 73 & 15.9 & 34 & 14.9 & 39 & 17 \\
\hline & Missing & 45 & 9.8 & 25 & 10.9 & 20 & 8.7 \\
\hline
\end{tabular}

TABLE 2 | Univariate and multivariate analysis of prognosis genes for TCGA CM.

\begin{tabular}{|c|c|c|c|c|c|c|}
\hline \multirow{2}{*}{$\begin{array}{l}\text { Training cohort } \\
\text { Genes }\end{array}$} & \multicolumn{3}{|c|}{ Univariate analysis } & \multicolumn{3}{|c|}{ Multivariate analysis } \\
\hline & coef & $\mathrm{HR}(95 \% \mathrm{Cl})$ & $P$ & coef & $\mathrm{HR}(95 \% \mathrm{Cl})$ & $P$ \\
\hline ADAMDEC1 & -0.098 & $0.906(0.848-0.969)$ & 0.004 & -0.099 & $0.906(0.845-0.971)$ & 0.005 \\
\hline D2S69E & -0.084 & 0.919 (0.847-0.998) & 0.043 & -0.089 & 0.915 (0.839-0.998) & 0.045 \\
\hline HSPA13 & -0.346 & $0.708(0.594-0.844)$ & 0.000 & -0.251 & $0.778(0.648-0.936)$ & 0.007 \\
\hline TRIM29 & 0.088 & $1.092(1.033-1.154)$ & 0.002 & 0.074 & $1.077(1.017-1.141)$ & 0.011 \\
\hline Validation cohort & & Univariate analysis & & & Multivariate analysis & \\
\hline Genes & coef & $\mathrm{HR}(95 \% \mathrm{Cl})$ & $P$ & coef & $\mathrm{HR}(95 \% \mathrm{Cl})$ & $P$ \\
\hline ADAMDEC1 & -0.102 & $0.903(0.852-0.958)$ & 0.000 & -0.113 & $0.893(0.834-0.956)$ & 0.001 \\
\hline D2S69E & -0.099 & $0.905(0.843-0.971)$ & 0.006 & -0.092 & $0.090(0.844-0.986)$ & 0.021 \\
\hline HSPA13 & -0.232 & $0.793(0.637-0.987)$ & 0.038 & -0.227 & $0.797(0.641-0.991)$ & 0.042 \\
\hline TRIM29 & 0.112 & $1.119(1.066-1.174)$ & 0.000 & 0.101 & $1.106(1.052-1.164)$ & 0.000 \\
\hline Total & & Univariate analysis & & & Multivariate analysis & \\
\hline Genes & coef & $\mathrm{HR}(95 \% \mathrm{Cl})$ & $P$ & coef & $\mathrm{HR}(95 \% \mathrm{Cl})$ & $P$ \\
\hline ADAMDEC1 & -0.101 & 0.905 (0.866-0.945) & 0.000 & -0.108 & $0.898(0.857-0.940)$ & 0.000 \\
\hline D2S69E & -0.091 & $0.913(0.865-0.963)$ & 0.000 & -0.093 & $0.911(0.862-0.964)$ & 0.000 \\
\hline HSPA13 & -0.284 & $0.753(0.657-0.864)$ & 0.000 & -0.264 & 0.768 (0.669-0.882) & 0.000 \\
\hline TRIM29 & 0.102 & $1.108(1.068-1.148)$ & 0.000 & 0.091 & 1.095 (1.055-1.136) & 0.000 \\
\hline
\end{tabular}

the low-risk group with the median OS time of 56.8 months had a higher survival ratio $(\mathrm{P}<0.001$; Figure 3A).

Furthermore, we analyzed the correlation between risk score and clinicopathological characteristics, which showed that high risk score was positively associated with elder age, ulceration, and breslow depth. Patients who received chemotherapy and radiotherapy prone to low-risk (Figures 3B-J).

\section{Stratification Analysis}

According to K-M analysis, CM patients with high risk score and larger breslow depth had the worst outcomes (Figure 4A), and $\mathrm{CM}$ patients with the ulceration and high- risk score had a shorter survival time than those with the non-ulceration group (Figure 4B). Furthermore, high risk score was also associated with poor prognosis in CM patients treated with chemotherapy 
J
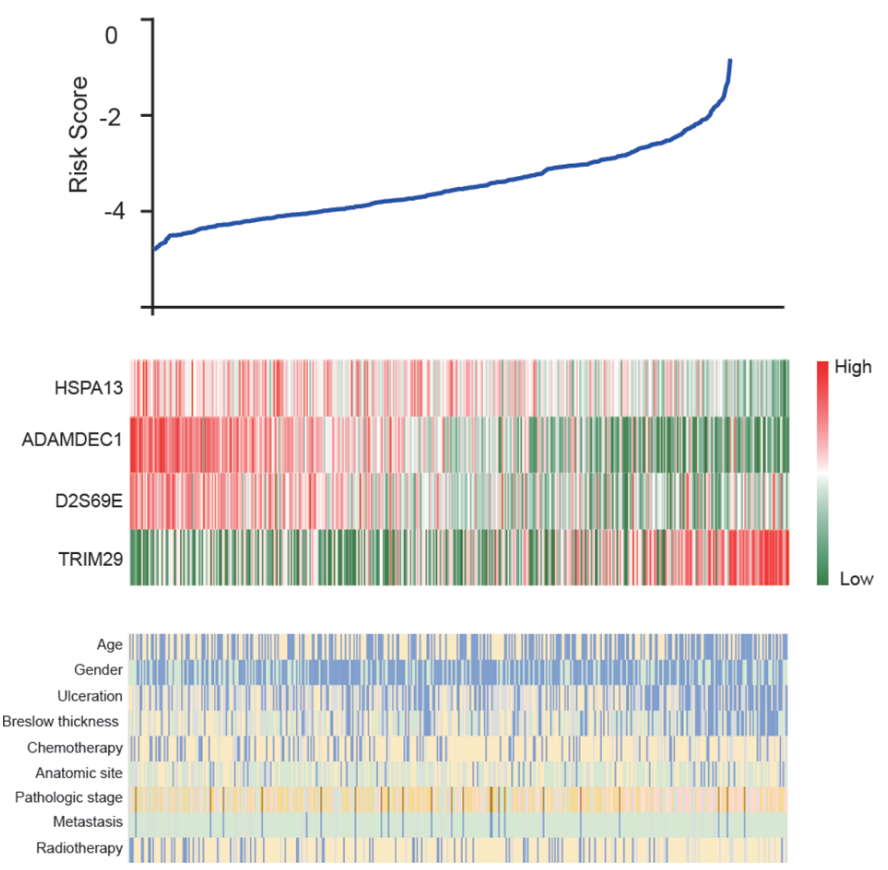

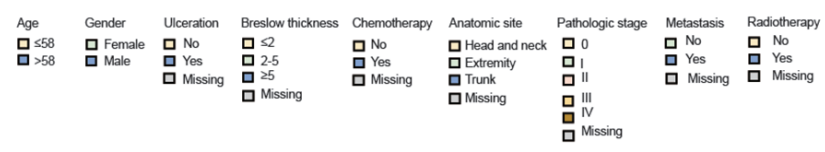

A

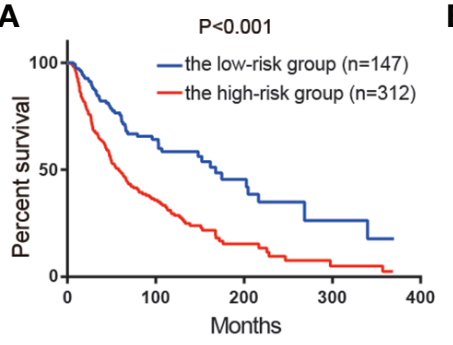

D

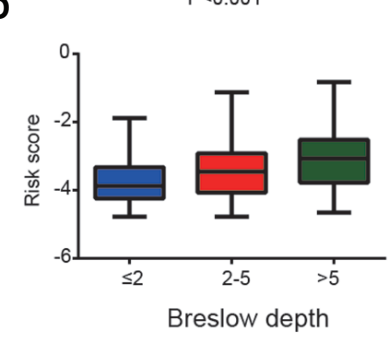

G

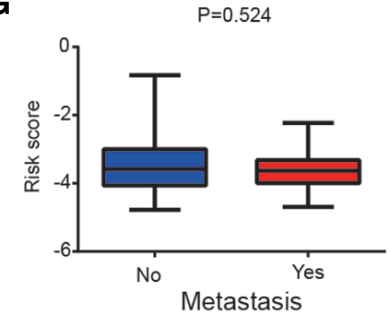

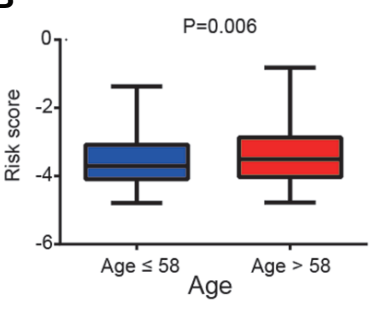

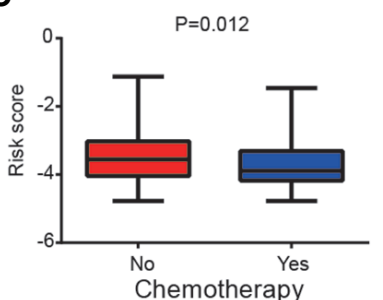

E

$P=0.385$

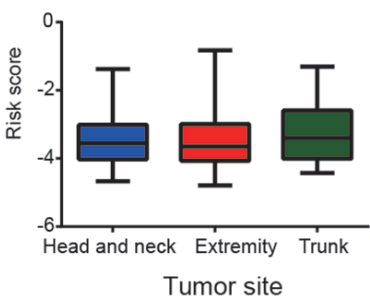

H

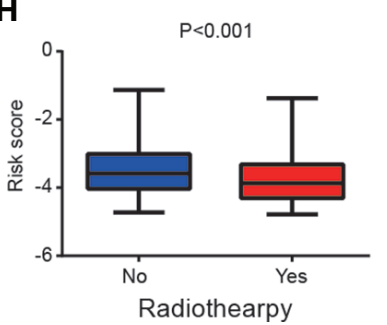

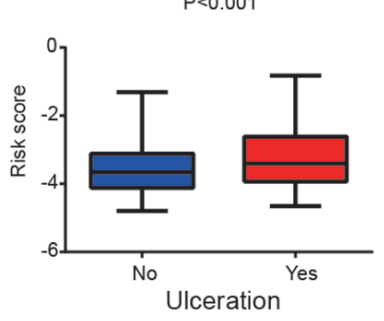

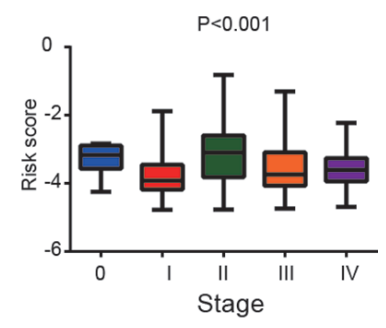

FIGURE 3 | The four-gene signature-derived risk score. (A) Kaplan-Meier overall survival analysis among TCGA CM patients stratified by risk score. Association between the risk model and different clinical characteristics. (B) Age. (C) Chemotherapy. (D) Breslow depth. (E) Tumor site. (F) Ulceration. (G) Metastasis. (H) Radiotherapy. (I) Stage. (J) The value of risk score (top), The corresponding expression of four genes (middle), and the associated clinicopathological parameters (bottom). 
A

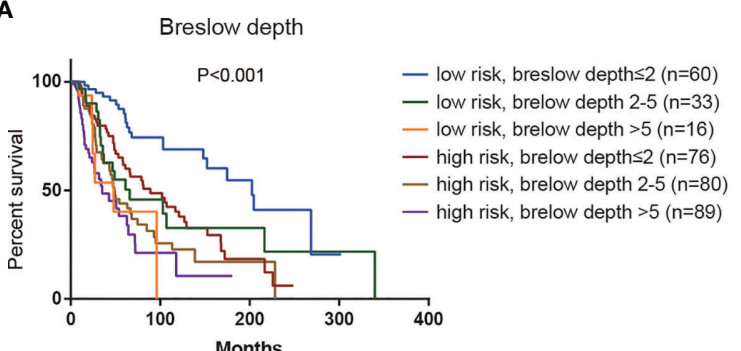

C

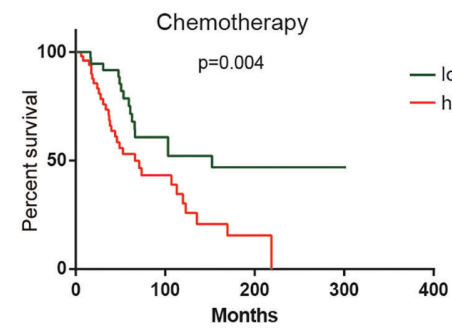

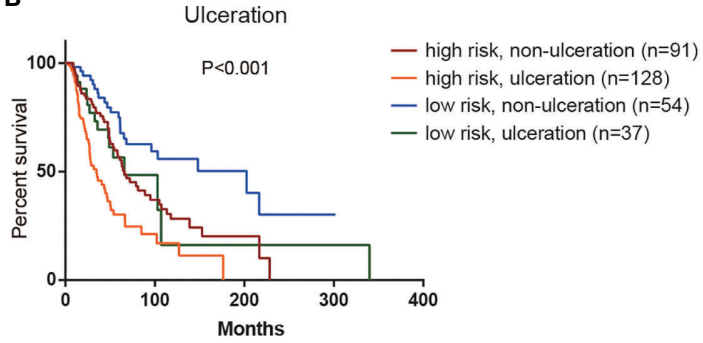

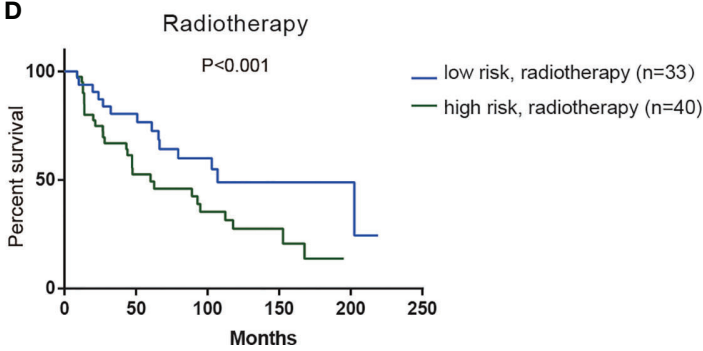

FIGURE 4 | Stratification analysis. Kaplan-Meier overall survival analysis of TCGA CM patients stratified by risk scores combined with (A) Breslow depth. (B). Ulceration. (C) Chemotherapy. (D) Radiotherapy.

or radiotherapy (Figures $4 \mathrm{C}, \mathrm{D}$ ), indicating that the risk score could predict the therapeutic reaction.

\section{Survival Predictive Model Based on Clinical Factors Alone or Their Combination With Risk Score}

We constructed a survival prediction model to identify whether risk score in the presence of clinical factors to better discriminate survival of CM patients. Compared with the model with clinical factors alone, the model with addition of the risk score improved the sensitivity and specificity of discriminating 1-year (AUC, 0.57 to 0.66 , Figure 5A), 3-year (AUC, 0.61 to 0.66 , Figure 5B), and 5-year survival (AUC, 0.61 to 0.70 , Figure 5C). When the model had both the risk score and clinical factors, its predictive ability for survival was greater [Concordance index (C-index) = $0.66]$ than that with clinical factors alone $(\mathrm{C}$-index $=0.59)$.

\section{External Validation of the Model in GSE65904}

GSE65904 dataset was used to validate the prediction performance of the model and each patient's risk score was calculated according to the formula of the model. All patients were divided into two groups: the high-risk group and the lowrisk group by the optimal cut-off value of risk score. The K-M curve revealed significant difference in overall survival between groups in GSE65904. High-risk group had markedly poorer outcome than low-risk group with $\mathrm{P}<0.05$ in Figure 6.

\section{The Association Between Prognosis Related Gene and Immune Markers}

In order to detect the correlation between prognosis related gene and the immune infiltration level, we concentrated particularly on the relationship between prognosis related gene and immune
A

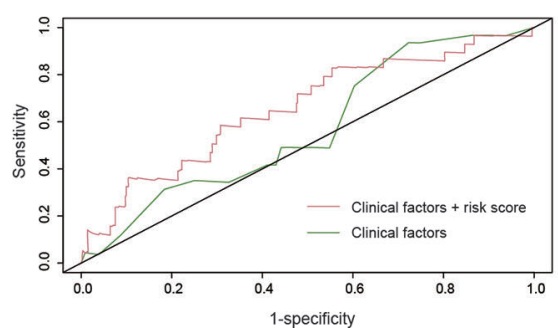

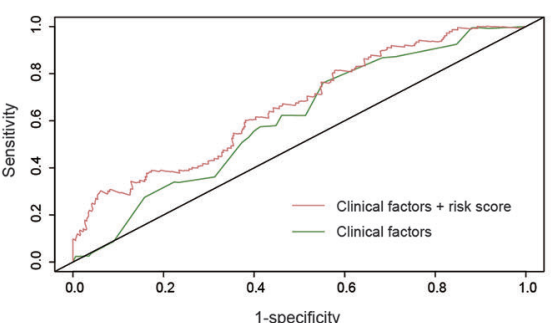

C

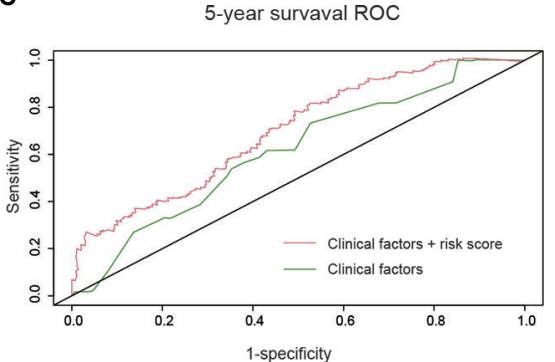

FIGURE 5 | Survival prediction model under the comparison of clinical factors versus the combination of risk score and clinical factors. (A) One-year survival receiver operating characteristic curves (ROC); (B) 3-year survival ROC; (C) 5-year survival ROC. 


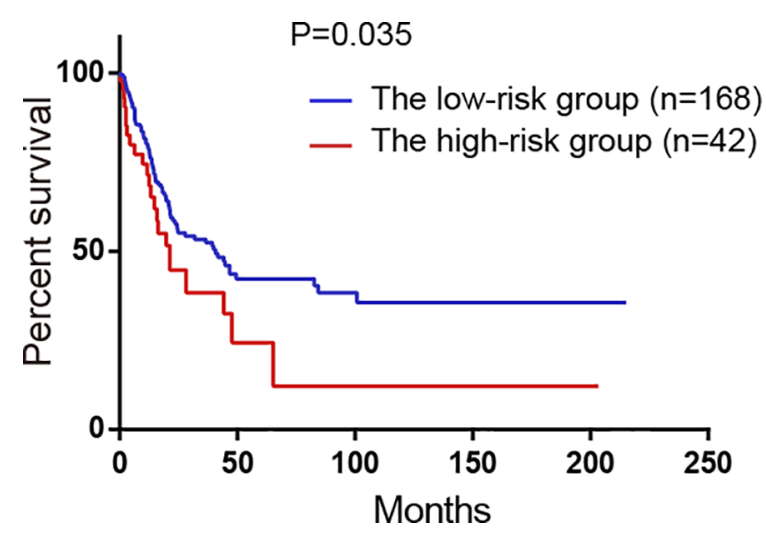

FIGURE 6 | Survival analysis of the high-risk group and the low-risk group divided by the model in GSE65904 validation set. All 214 patients were classified into two groups: the high-risk group and the low-risk group by the optimal cut-off value of risk scores.

markers of various immune cells in CM using the TIMER database. There was a positive correlation between ADAMDEC1 expression and the dendritic cell (Cor $=0.67, p=4.72 \mathrm{e}-59$ ), neutrophils (Cor $=0.652, p=3.99 \mathrm{e}-56)$, CD8+

$\mathrm{T}$ cells (Cor $=0.572, p=2.05 \mathrm{e}-39$ ), macrophages (Cor $=0.404$, $p=3.12 \mathrm{e}-19), \mathrm{CD} 4+\mathrm{T}$ cells (Cor $=0.385, p=3.45 \mathrm{e}-17$ ), B cells (Cor $=0.371, p=4.51 \mathrm{e}-16)$. Similar results were obtained for GNLY and HSPA13 (Figures 7A-C). While, the correlation between TRIM29 and immune infiltration is not obvious (Figure 7D). According to K-M analysis, high ADAMDEC1, HSPA13, and GNLY expression was significantly correlated with better prognosis, while high TRIM29 expression was markedly correlated with poor prognosis (Figures $7 \mathbf{E}-\mathbf{H}$ ).

\section{DISCUSSION}

In present study, we selected and constructed a four-gene based risk score model for CM. We analyzed GSE7553, GSE46517, and GSE15605 data sets, 103 DEGs were identified and selected. Subsequently, univariate and multivariate COX regression were employed for the key genes. Fourteen genes (GNLY, DFNA28, ADAMDEC1, ALOXE3, EFNA3, EPN3, EVPL, FERMT1, HSPA13, JAG2, RAPGEFL1, SULT2B1, TGM3, and TRIM29) were finally identified to be the prognostic genes. Here we adopted stepwise multivariate Cox regression analysis to select the best performance efficacy predictive model with the lowest AIC value. Finally, a fourgene based model including GNLY, ADAMDEC1, HSPA13, and TRIM29 was successfully developed.

Furthermore, in order to evaluate the prognostic significance of the new risk model, we performed log-rank test and the ROC curve analysis to investigate association between the model and clinical parameters. As we expected, the high-risk cohort was correlated with poor outcome and was tend to larger breslow depth and ulceration.

For our prognosis related genes, researchers have revealed that some of them may be crucial in cancer development, including CM. For instance, ALOXE3, which encodes arachidonate lipoxygenase3, can serve as a potential predictive biomarker for colon adenocarcinoma patients. Low expression of ALOXE3 had a favorable prognosis of COAD (11). Gómez-Maldonado et al. identified EFNA3, a member of the ephrin type A ligands, is induced by hypoxia-inducible factor in human tumors and this induction is predictive of poor prognosis and increased risk of metastasis in breast cancer patients (12). EPN3 expression is upregulated in wounded epithelial tissues and it can drive breast tumorigenesis by increasing E-cadherin endocytosis, EPN3 is overexpressed in $40 \%$ of breast cancers and its overexpression is an independent predictor of distant metastasis $(13,14)$.. Envoplakin (EVPL) is a protein component of desmosomes and the DNA variant in intron of EVPL (rs2071194) has been found associated with papillary and follicular thyroid cancer risk (15). FERMT1, as an oncogene, promotes the degradation of $\mathrm{I} \kappa \mathrm{B} \alpha$, thereby activating NF- $\mathrm{KB}$ signaling and promoting gastric cancer (16). JAG2 is one of Notch ligands, which recently appear to exert various carcinogenesis. JAG2 expression significantly correlates with angiogenic processes and vascular development in breast cancer, and is induced at the transcriptional level in hypoxic tumor cells. The oncogene c-myc can also modulate JAG2 expression under hypoxic conditions (17). In 2013, Takahashi et al. reported that RAPGEFL1 was highly methylated in some ESCC cell lines and RAPGEFL1 could regulate by most miRNAs. Therefore, RAPGEFL1 may be the potential pathogenic genes for ESCC (18). TGM3 could affect epithelial-mesenchymal transition, play an essential role in tumorigenesis and progression. It might serve as a useful biomarker and potential therapeutic target for hepatocellular carcinoma treatment (19).

Several genes in our risk model had been investigated in immune response. TRIM29, a member of the tripartite interaction motif (TRIM) family of proteins, functions as a negative regulator of innate immune response. Studies have shown that knockdown of TRIM29 in airway epithelial cells enhances type I interferon production (20). TRIM29 is also recognized as an oncogene, and elevated gene expression in multiple tumors such as colorectal cancer and bladder cancer and so on (21). But the function of TRIM29 in cutaneous melanoma remained still unknown. Elizabeth et al. discovered that ADAMDEC1, an orphan ADAM-like metalloprotease, is expressed in the immune system, by dendritic cells and macrophages. In vitro, the expression of ADAMDEC1 was significantly elevated in M1 but not M2 macrophages. More research is needed to determine the associations between ADAMDEC1 and immune response and associations with survival for cancers (22). Granulysin (GNLY) is a cytolytic apoptotic molecule highly expressed in activated immune cells, particularly human cytotoxic T lymphocytes (CTLs) and natural killer (NK) cells (23). GNLY functions as a lytic molecule to carry out lysis or apoptosis product in target cells, including tumor 
A

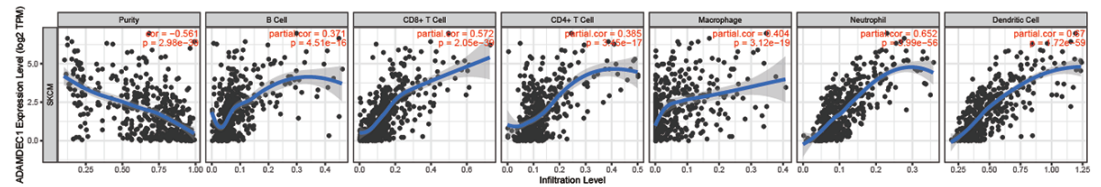

B

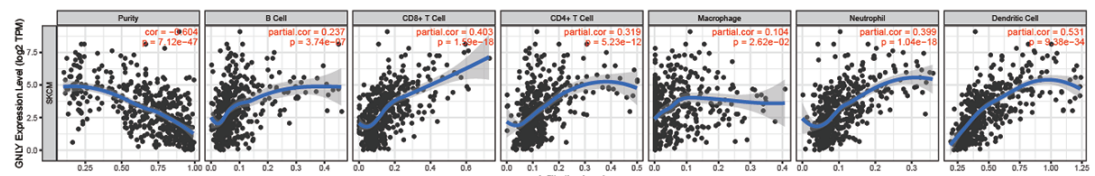

C

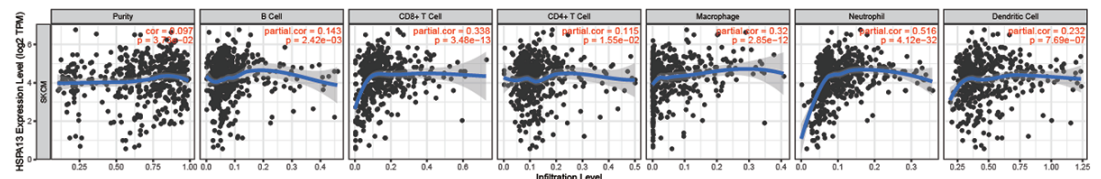

D

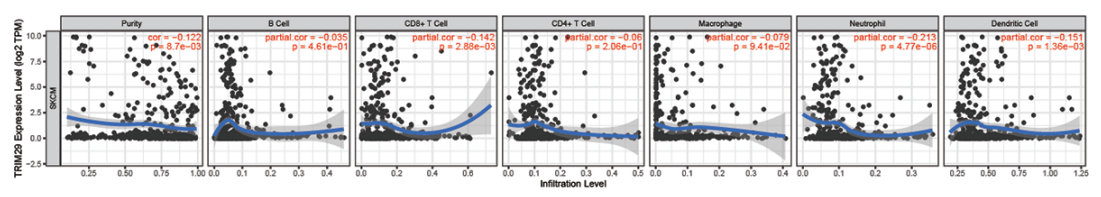

E

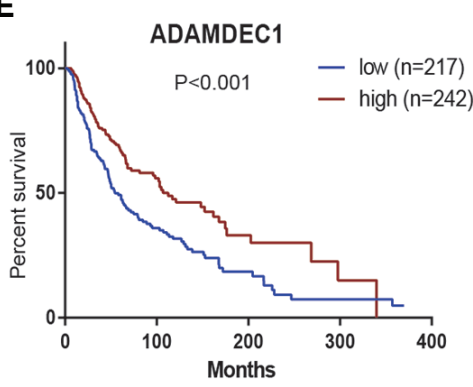

G

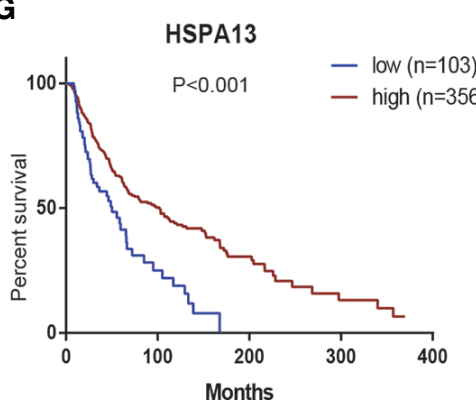

F

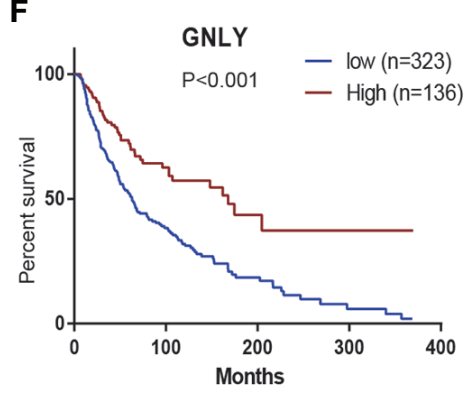

H

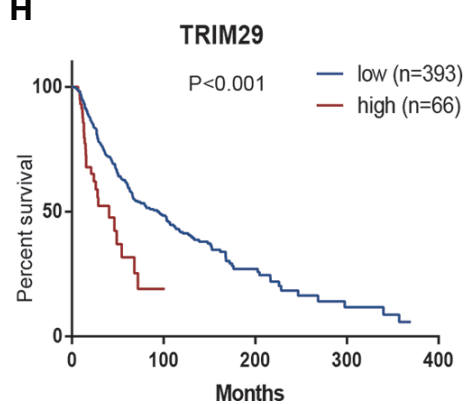

FIGURE 7 | The correlation between prognostic genes expression and immune cell infiltration in CM (TIMER database). The correlation between the abundance of immune cell and the expression of (A) ADAMDEC1; (B) GNLY; (C) HSPA13; (D) TRIM29. Prognostic values of (E) ADAMDEC1, (F) GNLY, (G) HSPA13, (H) TRIM29 in TCGA CM. 
cells or cells infected by pathogens. GNLY can also activate antigen-presenting cells through TLR4 (24). Multiple publications have confirmed the anti-tumor activity of GNLY (25-29). Ya-Wen reported that the serum level of GNLY was negatively correlated with the proliferation of transplanted tumor cells in HIS mice (30). All gene in this risk model are firstly studied in cutaneous melanoma.

To sum up, our research results indicate that the four-gene prognostic model is a reliable tool for predicting the overall survival of CM, it may be useful for guiding therapeutic strategies to improve the clinical outcome of melanoma patients. The lowrisk group should avoid some unnecessary treatment to reduced drug toxicities, and high-risk group can receive other intensive treatment. For clinical application, more clinical studies are needed to further verify the prognostic and predictive significance of the risk score model.

\section{CONCLUSIONS}

In conclusion, the new risk score system functions well in predicting the prognosis and treatment response in $\mathrm{CM}$ patients, with the potential to optimize treatment options. More studies are needed to explore the biological function of

\section{REFERENCES}

1. Carr S, Smith C, Wernberg J. Epidemiology and Risk Factors of Melanoma. Surg Clin North Am (2020) 100:1-12.

2. Rebecca VW, Somasundaram R, Herlyn M. Pre-clinical modeling of cutaneous melanoma. Nat Commun (2020) 11(1):2858. doi: 10.1038/ s41467-020-15546-9

3. de Assis LVM, Moraes MN, Castrucci AML. The molecular clock in the skin, its functionality, and how it is disrupted in cutaneous melanoma: a new pharmacological target? Cell Mol Life Sci (2019) 76(19):3801-26. doi: 10.1007/ s00018-019-03183-5

4. Siegel RL, Miller KD, Jemal A. Cancer statistics, 2015. CA Cancer J Clin (2015) 65(1):5-29. doi: 10.3322/caac.21254

5. Hartman RI, Lin JY. Cutaneous Melanoma-A Review in Detection, Staging, and Management. Hematol Oncol Clin North Am (2019) 33(1):25-38. doi: 10.1016/j.hoc.2018.09.005

6. Balch CM, Gershenwald JE, Soong SJ, Thompson JF, Atkins MB, Byrd DR, et al. Final version of 2009 AJCC melanoma staging and classification. J Clin Oncol (2009) 27(36):6199-206. doi: 10.1200/JCO.2009.23.4799

7. Bertoli G, Cava C, Castiglioni I. MicroRNAs: New Biomarkers for Diagnosis, Prognosis, Therapy Prediction and Therapeutic Tools for Breast Cancer. Theranostics (2015) 5(10):1122-43. doi: 10.7150/thno.11543

8. Sparano JA, Gray RJ, Makower DF, Pritchard KI, Albain KS, Hayes DF, et al. Adjuvant Chemotherapy Guided by a 21-Gene Expression Assay in Breast Cancer. N Engl J Med (2018) 379(2):111-21. doi: 10.1056/NEJMoa1804710

9. Daoud AZ, Mulholland EJ, Cole G, McCarthy HO. MicroRNAs in Pancreatic Cancer: biomarkers, prognostic, and therapeutic modulators. BMC Cancer (2019) 19(1):1130. doi: 10.1186/s12885-019-6284-y

10. Albain KS, Barlow WE, Shak S, Hortobagyi GN, Livingston RB, Yeh IT, et al. Prognostic and predictive value of the 21-gene recurrence score assay in postmenopausal women with node-positive, oestrogen-receptorpositive breast cancer on chemotherapy: a retrospective analysis of a randomised trial. Lancet Oncol (2010) 11(1):55-65. doi: 10.1016/S1470-2045 (09)70314-6

11. Ruan GT, Gong YZ, Zhu LC, Gao F, Liao XW, Wang XK, et al. The Perspective of Diagnostic and Prognostic Values of Lipoxygenases mRNA these four genes in CM progression and to further verify the prognostic value of the model for clinical practice.

\section{DATA AVAILABILITY STATEMENT}

The original contributions presented in the study are included in the article/supplementary material. Further inquiries can be directed to the corresponding author.

\section{AUTHOR CONTRIBUTIONS}

This work was carried out in collaboration with all authors. MW designed the theme of the article. XT wrote and XQ reviewed the article. All authors contributed to the article and approved the submitted version.

\section{ACKNOWLEDGMENTS}

We thank Yuhu Xin, Ping Zhang, and Na Wang for their support over the past years.

Expression in Colon Adenocarcinoma. Onco Targets Ther (2020) 13:9389405. doi: 10.2147/OTT.S251965

12. Gomez-Maldonado L, Tiana M, Roche O, Prado-Cabrero A, Jensen L, Fernandez-Barral A, et al. EFNA3 long noncoding RNAs induced by hypoxia promote metastatic dissemination. Oncogene (2015) 34(20):260920. doi: $10.1038 /$ onc. 2014.200

13. Spradling KD, McDaniel AE, Lohi J, Pilcher BK. Epsin 3 is a novel extracellular matrix-induced transcript specific to wounded epithelia. J Biol Chem (2001) 276(31):29257-67. doi: 10.1074/jbc.M101663200

14. Schiano Lomoriello I, Giangreco G, Iavarone C, Tordonato C, Caldieri G, Serio G, et al. A self-sustaining endocytic-based loop promotes breast cancer plasticity leading to aggressiveness and pro-metastatic behavior. Nat Commun (2020) 11(1):3020. doi: 10.1038/s41467-020-16836-y

15. Sigurdson AJ, Brenner AV, Roach JA, Goudeva L, Muller JA, Nerlich K, et al. Selected single-nucleotide polymorphisms in FOXE1, SERPINA5, FTO, EVPL, TICAM1 and SCARB1 are associated with papillary and follicular thyroid cancer risk: replication study in a German population. Carcinogenesis (2016) 37(7):677-84. doi: 10.1093/carcin/bgw047

16. Fan H, Zhang S, Zhang Y, Liang W, Cao B. FERMT1 promotes gastric cancer progression by activating the NF-kappaB pathway and predicts poor prognosis. Cancer Biol Ther (2020) 21(9):815-25. doi: 10.1080/ 15384047.2020.1792218

17. Pietras A, von Stedingk K, Lindgren D, Pahlman S, Axelson H. JAG2 induction in hypoxic tumor cells alters Notch signaling and enhances endothelial cell tube formation. Mol Cancer Res (2011) 9(5):626-36. doi: 10.1158/1541-7786.MCR-10-0508

18. Takahashi T, Matsuda Y, Yamashita S, Hattori N, Kushima R, Lee YC, et al. Estimation of the fraction of cancer cells in a tumor DNA sample using DNA methylation. PloS One (2013) 8(12):e82302. doi: 10.1371/journal.pone.0082302

19. Hu JW, Yang ZF, Li J, Hu B, Luo CB, Zhu K, et al. TGM3 promotes epithelialmesenchymal transition and hepatocellular carcinogenesis and predicts poor prognosis for patients after curative resection. Dig Liver Dis (2020) 52(6):66876. doi: 10.1016/j.dld.2019.10.010

20. Xing J, Weng L, Yuan B, Wang Z, Jia L, Jin R, et al. Identification of a role for TRIM29 in the control of innate immunity in the respiratory tract. Nat Immunol (2016) 17(12):1373-80. doi: 10.1038/ni.3580 
21. Sun J, Zhang T, Cheng M, Hong L, Zhang C, Xie M, et al. TRIM29 facilitates the epithelial-to-mesenchymal transition and the progression of colorectal cancer via the activation of the Wnt/beta-catenin signaling pathway. $J$ Exp Clin Cancer Res (2019) 38(1):104. doi: 10.1186/s13046-019-1098-y

22. Bates EE, Fridman WH, Mueller CG. The ADAMDEC1 (decysin) gene structure: evolution by duplication in a metalloprotease gene cluster on chromosome 8p12. Immunogenetics (2002) 54(2):96-105. doi: 10.1007/s00251-002-0430-3

23. Vujaklija DV, Gulic T, Sucic S, Nagata K, Ogawa K, Laskarin G, et al. First trimester pregnancy decidual natural killer cells contain and spontaneously release high quantities of granulysin. Am J Reprod Immunol (2011) 66(5):36372. doi: 10.1111/j.1600-0897.2011.01015.x

24. Tewary P, Yang D, de la Rosa G, Li Y, Finn MW, Krensky AM, et al. Granulysin activates antigen-presenting cells through TLR4 and acts as an immune alarmin. Blood (2010) 116(18):3465-74. doi: 10.1182/blood-2010-03-273953

25. Clayberger C, Krensky AM. Granulysin. Curr Opin Immunol (2003) 15 (5):560-5. doi: 10.1016/S0952-7915(03)00097-9

26. Lin J, Huang Y, Zhang L, Tang W, Li X, Wang X, et al. Evaluation of serum granulysin as a potential biomarker for nasopharyngeal carcinoma. Clin Chim Acta (2016) 454:72-6. doi: 10.1016/j.cca.2015.12.035

27. Aporta A, Catalan E, Galan-Malo P, Ramirez-Labrada A, Perez M, Azaceta G, et al. Granulysin induces apoptotic cell death and cleavage of the autophagy regulator Atg5 in human hematological tumors. Biochem Pharmacol (2014) 87(3):410-23. doi: 10.1016/j.bcp.2013.11.004
28. Jiang W, Zhu D, Wang C, Zhu Y. An immune relevant signature for predicting prognoses and immunotherapeutic responses in patients with muscle-invasive bladder cancer (MIBC). Cancer Med (2020) 9(8):2774-90. doi: $10.1002 /$ cam4.2942

29. Saini RV, Wilson C, Finn MW, Wang T, Krensky AM, Clayberger C. Granulysin delivered by cytotoxic cells damages endoplasmic reticulum and activates caspase-7 in target cells. J Immunol (2011) 186(6):3497-504. doi: 10.4049/jimmunol.1003409

30. Hsiao YW, Lai TC, Lin YH, Su CY, Lee JJ, Liao AT, et al. Granulysin expressed in a humanized mouse model induces apoptotic cell death and suppresses tumorigenicity. Oncotarget (2017) 8(48):83495-508. doi: 10.18632/ oncotarget.11473

Conflict of Interest: The authors declare that the research was conducted in the absence of any commercial or financial relationships that could be construed as a potential conflict of interest.

Copyright (c) 2021 Tong, Qu and Wang. This is an open-access article distributed under the terms of the Creative Commons Attribution License (CC BY). The use, distribution or reproduction in other forums is permitted, provided the original author(s) and the copyright owner(s) are credited and that the original publication in this journal is cited, in accordance with accepted academic practice. No use distribution or reproduction is permitted which does not comply with these terms. 